\title{
Investigating confounders of the association between survival and adjuvant radiation therapy after breast conserving surgery in a sample of elderly breast Cancer patients in Appalachia
}

Fabian Camacho ${ }^{1 *}$, Roger Anderson ${ }^{1}$ and Gretchen Kimmick ${ }^{2}$

\begin{abstract}
Background: To explain the association between adjuvant radiation therapy after breast conserving surgery (BCS RT) and overall survival (OS) by quantifying bias due to confounding in a sample of elderly breast cancer beneficiaries in a multi-state region of Appalachia.

Methods: We used Medicare claims linked registry data for fee-for-service beneficiaries with AJCC stage I-III, treated with BCS, and diagnosed from 2006 to 2008 in Appalachian counties of Kentucky, Ohio, North Carolina, and Pennsylvania. Confounders of BCS RT included age, rurality, regional SES, access to radiation facilities, marital status, Charlson comorbidity, Medicaid dual status, institutionalization, tumor characteristics, and surgical facility characteristics. Adjusted percent change in expected survival by BCS RT was examined using Accelerated Failure Time (AFT) models. Confounding bias was assessed by comparing effects between adjusted and partially adjusted associations using a fully specified structural model.

Results: The final sample had 2675 beneficiaries with mean age of 75, with 81\% 5-year survival from diagnosis. Unadjusted percentage increase in expected survival was 2.75 times greater in the RT group vs. non-RT group, with 5 -year survival of $85 \%$ vs $60 \%$; fully adjusted percentage increase was 1.70 times greater, with 5 -year rates of $83 \%$ vs $71 \%$. Quantification of incremental confounding showed age accounted for $71 \%$ of the effect reduction, followed by tumor features (12\%), comorbidity (10\%), dual status(10\%), and institutionalization (8\%). Adjusting for age and tumor features only resulted in only $4 \%$ bias from fully adjusted percent change (70\% change vs 66\%).

Conclusion: Quantification of confounding aids in determining covariates to adjust for and in interpreting raw associations. Substantial confounding was present (60\% of total association), with age accounting for the largest share (71\%); adjusting for age plus tumor features corrected for most of the confounding (4\% bias). The direct effect of BCS RT on OS accounted for $40 \%$ of the total association.
\end{abstract}

\footnotetext{
* Correspondence: fcamacho@virginia.edu

${ }^{1}$ Department of Public Health Sciences, University of Virginia, Charlottesville,

VA 22903, USA

Full list of author information is available at the end of the article
}

C The Author(s). 2019 Open Access This article is distributed under the terms of the Creative Commons Attribution 4.0 International License (http://creativecommons.org/licenses/by/4.0/), which permits unrestricted use, distribution, and reproduction in any medium, provided you give appropriate credit to the original author(s) and the source, provide a link to the Creative Commons license, and indicate if changes were made. The Creative Commons Public Domain Dedication waiver (http://creativecommons.org/publicdomain/zero/1.0/) applies to the data made available in this article, unless otherwise stated. 


\section{Background}

Radiation therapy (RT) after breast conserving surgery (BCS) in non-metastatic breast cancer (BC) patients has been shown in clinical studies to improve long term (> 10 years) survival [1-4], compared to BCS alone. Thus adjuvant $\mathrm{RT}$ has been a consistent recommendation in standard of care guidelines for Stage I-III patients undergoing BCS [5-7]. Analysis of registry data, at the population level, suggests even larger survival benefits of RT [8, 9] and detectable differences in short term survival $(<6$ years), which remains in certain subpopulations even after adjusting for relevant confounders [10-12].

A major challenge of the latter real-word or "effectiveness "studies is to explain the magnitude of the association of a recommended therapy, such as $\mathrm{RT}$, on survival apart from the influence of important confounding factors, which often include comorbidity, access to care, socio-economic status, and quality of care $[10,11]$. Such a quantification of confounding may be important in determining whether a covariate needs to be adjusted for in an analysis and aid in the interpretation of unadjusted associations [13].

In this study, we document the effect of adjuvant RT on survival to model and quantify the magnitude of the benefit from treatment that may be due to significant confounding. Methods for quantifying confounding have been well-documented which enable investigation of confounding bias [13] and which are implemented in this paper. The study population is a sample of a mostly elderly population residing in Appalachian counties of 4 states (Pennsylvania, Ohio, Kentucky, North Carolina) as defined by the Appalachian Regional Commission and chosen to capture the breadth of the Appalachian region. This geographical region has higher cancer incidence and mortality rates [14-16], applicable to breast cancer mortality as well [17], with heterogeneous economic diversity [18], poor health care accessibility $[18,19]$ significant medically underserved pockets [18], and is distinct from regions from studies using SEER (Surveillance, Epidemiology, and End Results Program)-Medicare linked databases [20-22].

\section{Methods}

\section{Study population}

Female sample beneficiaries with breast cancer and diagnosed from 2006 to 2008 resided in the four state region of Appalachia defined by the Appalachian Regional Commission (see Additional file 1 map, section 3). Beneficiary characteristics were extracted from each state cancer registry. Beneficiaries were then linked to their corresponding Medicare claims from 2005 to 2009 by matching social security number, birth date, and gender. Conforming to published inclusion criteria [23], beneficiaries were restricted to having diagnostically confirmed breast cancers, fee for service (FFS) continuous Medicare enrollment 1 year after and before diagnosis, a first cancer diagnosis, no multiple/concurrent cancers within 90 days, 1 year survival after cancer diagnosis, AJCC (American Joint Committee on Cancer) stage I-III, and BCS during 6 months after diagnosis.

\section{Study variables}

Overall survival was derived from a composite of updated registry NDI (National Death Index) survival and Medicare beneficiary death date updated until Dec 2014. Identification of BCS was based on registry primary site surgery variable supplemented by Medicare claims using a study-specific algorithm [23]. Codes used included registry site-specific codes (10-20), ICD-9-CM procedure codes 85.20-8.23, 86.25, and HCPCS/CPT codes 19,120, 19,125, $19,126,19,160,19,162,19,301,19,302$ [23]. Identification of Adjuvant RT was based on a registry radiation sequence variable supplemented by Medicare claims using look-up tables provided by the NCI (National Cancer Institute) [24]. RT was assumed given if radiation codes appeared for 15 days after surgery during the year after diagnosis.

Geographical variables included county level measures for rurality based on the US Department of Agriculture 2013 Beale codes [25] and an area deprivation index (Singh) based on 2000 census measures [26]. The number of hospitals/health care systems with radiation services within a 50 mile straight-line radius from patient residence was calculated as a measure of accessibility, as determined from AHA (American Hospital Association) annual 2010 survey database [27]. The 50 mile cut-off was chosen based on choosing the cut-off with strongest prediction of RT delivery, based on fitting a logistic regression with $\mathrm{RT}$ delivery rate as a function of successively increasing distance thresholds ranging from 25 to 100 miles in 5 mile increments.

Potential confounders included beneficiary comorbidity, enrollment in Medicaid during year after diagnosis, and being institutionalized. Comorbidity was based on a Charlson-Deyo score [28, 29] calculated during the year prior to diagnosis. Institutionalization was assessed if either at least two claims existed with shared living, nursing/custodial facility, or hospice, or beneficiary had more than 15 days in a skilled nursing facility during the year after diagnosis. Other confounders consisted of age at diagnosis, marital status (single vs married), AJCC Derived Stage (I,II, III), first cancer, grade, histology, positive lymph nodes, ER/PR (Estrogen/Progesterone) positive tumor, tumor size, total number of beds and therapeutic radiology services of surgical facility [23], facility accreditation with the COC (Commission of Cancer) obtained from a web site locator during 2011 [30], and surgical provider volume calculated from claims during the calendar year 2008.

Although RT after BCS has been viewed as guideline for all cases $[6,7]$, other authors suggest RT as optional for subgroups of elderly patients, such as patients jointly 
aged $\geq 70$ years, with ER/PR positive, $<2 \mathrm{~cm}$, and node negative tumors [23]. These recommendations are based on findings documenting lower benefit of RT in these subgroups [31, 32] . As a result, an indicator for optional RT was included as a potential confounder.

\section{Statistical methods}

The primary effect of interest is percent change in expected survival time $(E[T])$ between survival for patients if they receive BCS-RT vs if they do not receive BCS-RT $(\delta=(\mathrm{E}[\mathrm{T}(1)]-\mathrm{E}[\mathrm{T}(0)]) / \mathrm{E}[\mathrm{T}(0)])$. Confounding bias is defined as the difference $(\Delta)$ between the marginal association $\delta_{\mathrm{m}}(\mathrm{E}[\mathrm{T} \mid \mathrm{X}=1]-\mathrm{E}[\mathrm{T} \mid \mathrm{X}=0]) / \mathrm{E}[\mathrm{T} \mid \mathrm{X}=0]$ and $\delta$ [13]. Incremental confounding (IC) is the decomposition of this bias into contributions from each confounder.

Several methods have been suggested to estimate $\delta, \Delta$, and IC, which include standardization and Inverse Probability of Treatment Weights (IPTW) [13]. However, a regression based approach, is an alternative. Advantages of this method include its similarity to traditional regression techniques. Also, $\delta, \Delta$, and IC can be calculated under the presence of interactions with the main exposure. Doubly robust approaches combining IPTW and regression simultaneously may further protect against misspecification [33]. However, the statistical properties in the context of censored survival data and confounding quantification remains to be studied and is not covered in this paper.

Directed Acyclic Graphs (DAGS) (Fig. 1) are first presented to clarify the causal structure between variables. Figure 1 a describes the assumed causal network, where the topmost layer of nodes represents confounder variables ordered from antecedent to subsequent in a causal chain, and the bottom layer describes nodes representing the exposure (BCS-RT) and outcome (Survival). The quantification method successively conditions on confounders and uses the conditional associations between exposureoutcome. Graphically, pathways leading through conditioned nodes cease to contribute to the association, allowing for progressive elimination of pathways until a direct causal effect can be estimated [34]. The method of successive conditioning is applicable even if latent variables affect or are affected by the confounders, as illustrated in Fig. 1 b. One exception is shown in Fig. 1 c, where latent variables simultaneously affect one of the confounders, leading to an additional non-causal bias. Similarly, omitting important confounders (Fig. $1 \mathrm{~d}$ ), results in biased effects.

Once the causal network has been considered, a parametric Accelerated Failure Time (AFT) model linking predictors to overall survival time was fit to the data. Although AFT models are a less applied alternative to the more popular Cox Proportional Hazards model $[35,36]$, an advantage is that log survival times are regressed directly on a linear combination of predictors, with an error term commonly following a log logistic, Weibull, or an exponential distribution. Percentage change in survival time compared to reference value can be calculated from the regression weights [37, 38]. Effects were considered statistically significant if False Discovery Rate (FDR) adjusted p-values were $<.05$ [39].

We sought to reduce several sources of misspecification bias for the full model. Estimation bias due to misspecification of the error distribution was addressed by choosing the model with lowest AIC (Akaike Information Criterion) [40]. A second source of bias exist if effect heterogeneity was present. Initially, a main effects AFT model was fit assuming the effect represents an average across covariate levels. Effect heterogeneity was then investigated through subgroup analysis, examining the effect of RT across all covariate levels; interaction terms were added to the main effects model if their separate interaction test $p$-values $<.05$. Other sources of bias concerned the possibility of misclassification error, which is not considered in this paper, and endogeneity bias, where the error term is correlated with the exposure. For the fully adjusted model, we expect the error term to be uncorrelated under the assumption that no a.

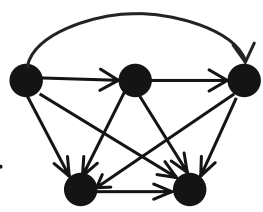

b.

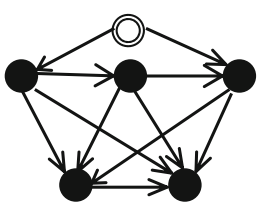

c.

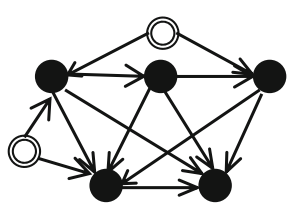

d.

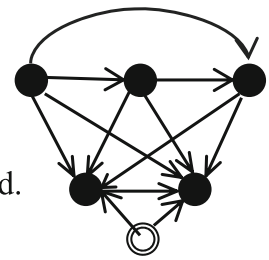

Fig. 1 Directed Acyclic Graphs for Confounding Quantification. Shows causal graphs under which quantification is applicable or not applicable 
residual confounding is present. Finally, non-informative censoring was assumed.

The next step consisted in estimating the effects $\delta, \delta_{\mathrm{m}}$, and partially adjusted effects $\delta_{\mathrm{Z}}$ used to assess incremental confounding. These quantities were estimated using estimates from the fully adjusted and partially adjusted models. The partially adjusted models retained the same specification as the fully adjusted model but without variables that were not adjusted for. More detail is provided in the Additional file 1, section 1. From these quantities, overall $\Delta$ and decomposition of $\Delta$ into increments were calculated, where each increment assessed the change in confounding bias. Confidence intervals were calculated by non-parametric bootstrapping (1000 resamples) with replacement and using percentile method to determine endpoints.

Model based survival curves after confounder adjustment were calculated using the "direct adjusted" method [41-43] which averaged predicted survival from the fully specified model at each time point for each patient. In addition, Kaplan Meier (KM) and unadjusted modelbased curves based on an unadjusted AFT model assessing the uncontrolled association between BCS-RT survival were calculated.

In order to treat missing data, multiple imputation (MI) was conducted using the method by White and Royston [44], which adds the cumulative hazard and censoring variable to the imputation model. Missing values were then imputed using the FCS (Fully Conditional Specification) method available in the SAS procedure MI, v9.3. To improve compatibility of the imputation model with the analysis model with interactions, the MI procedure was implemented separately on RT strata. In order to integrate MI into the calculation of confidence intervals, the data was bootstrapped first and MI was conducted for each bootstrap, as recommended in [45].

\section{Results}

The final sample consisted of 2675 beneficiaries. From Table 1 , the average age was 75 , most patients lived in metropolitan regions (59\%), average number of local radiation facilities was 5 , average Charlson score was 1.34 (standard deviation $=1.62$ ), only $15 \%$ where Medicaid duals, $6 \%$ were institutionalized, $70 \%$ were Stage I, $85 \%$ had first cancer tumors, $50 \%$ had moderately differentiated grade, $92 \%$ had Ductal/tubular histology, $85 \%$ were lymph node negative, $88 \%$ were ER/PR positive, average tumor size was $1.6 \mathrm{~cm}, 71 \%$ patients had surgical treatment in a COC designated facility, with average number of 339 beds, and $77 \%$ of such facilities offered radiation services. Lastly, $56 \%$ did not meet optional radiation therapy criteria.

Significant predictors of increased OS based on the fully adjusted main effect AFT model, using percent change (\% $\mathrm{CH})$ in expected survival as a measure of effect (Fig. 2), included receiving RT $(\% \mathrm{CH}=63)$, younger age (Q1,Q2,Q3 vs Q4, \%CH $=131,84,50)$, urban residence (vs rural, $\% \mathrm{CH}=47$ ), lower Charlson comorbidity $(0,1$ vs $2+$ vs $3, \% \mathrm{CH}=82,39)$, stage II (vs III, $\% \mathrm{CH}=$ $39)$, no prior cancer diagnosis $(\% \mathrm{CH}=23), \mathrm{ER} / \mathrm{PR}$ positive (vs negative, \% $\mathrm{CH}=31$ ), smaller tumor size $(\mathrm{Q} 1, \mathrm{Q} 2$, $\mathrm{Q} 3$ vs $\mathrm{Q} 4, \% \mathrm{CH}=83,60,49)$. Decreased OS was predicted by $\mathrm{OH}$ residence (vs $\mathrm{PA}, \% \mathrm{CH}=-18$ ), Medicaid Dual insurance status (\% $\mathrm{CH}=-20)$, and being institutionalized $(\% \mathrm{CH}=-51)$. Figure 3 shows results from the subgroup analysis. Variations, or heterogeneity, in the RT effect size, was detected for being institutionalized (\% $\mathrm{CH}$ Yes $=5$ vs $\mathrm{No}=54, p=.0160)$, stage $(\% \mathrm{CH} \mathrm{I}=33$, $\mathrm{II}=73, \mathrm{III}=107, p=.0016)$, first cancer $(\% \mathrm{CH}$ Yes $=58$ vs $\mathrm{No}=9, p=.0022$ ), lymph nodes (\% $\mathrm{CH}$ Positive $=78$ vs Negative $=43, p=.0506)$, tumor size $(\% \mathrm{CH} \mathrm{Q1}=36$, $\mathrm{Q} 2=33, \mathrm{Q} 3=27, \mathrm{Q} 4=90, p=.0005$ ), and optional RT (\% CH optional $\mathrm{Y}=24, N=74, p<.0001$ ).

Figure 4 shows the quantification of confounding based on comparing population averaged expected survival change $(\% \mathrm{CH})$, where survival is measured starting 1 year after diagnosis. The unadjusted $\% \mathrm{CH}$ for $\mathrm{RT}=$ $175 \%$, suggesting expected survival for those who receive $\mathrm{RT}$ is 2.75 times greater than those who were not treated with RT, whereas the fully adjusted \% $\mathrm{CH}$ based on a model with interactions from subgroup analysis was \% $\mathrm{CH}=70 \%$, or an expected survival 1.70 times greater with RT $(p<.0001)$. The difference between these two quantities was statistically significant $(\Delta=105$, bootstrap 95 CI\% 79,143).

When examining incremental confounding differences compared to total differences in annual survival using the default sequential order (left chart in Fig. 4), age accounts to $72 \%$ of the difference, there is a $5 \%$ increase due to adding geography, $5 \%$ reduction from marital status, $10 \%$ reduction from comorbidity, $10 \%$ reduction due to adding dual Medicaid/Medicare status, $8 \%$ reduction due to adding institutionalization, $12 \%$ reduction due to adding tumor features, and $11 \%$ increase by adding the remaining features. With the exception of institutionalization, all successive confounding differences were significant based on overlap of the $95 \%$ bootstrap confidence intervals with zero.

When confounders are separately added after adjusting for age (right chart in Fig. 4), all except for geography and 'other' contribute towards bias reduction. These include marital status (76\% reduction) comorbidity (84\%), dual (88\%), institutionalization (90\%), and tumor features $(103 \%)$. In particular, adding age plus tumor features only results in an \% change effect of $66.4 \%$ which, when compared to the fully adjusted effect of 69.5 , is off by a $4 \%$ bias.

Figure 5 shows KM and AFT model-based unadjusted/ adjusted survival curves stratified by RT and no RT 
Table 1 Characteristics and Predictors of Adjuvant Radiation Therapy ( $N=2675)$

\begin{tabular}{|c|c|c|c|}
\hline Overall & N (\%) & & $\mathrm{N}(\%)$ \\
\hline Age Quartile & & Grade $^{\S}$ & $\mathrm{N}_{\text {miss }}=158$ \\
\hline Q1 [ $\leq 69]$ & $700(26.2)$ & Well differentiated & $712(28.3)$ \\
\hline Q2 [70-75] & $701(26.2)$ & Moderately differentiated & $1203(47.8)$ \\
\hline Q3 [76-80] & $609(22.8)$ & Poor/Undifferentiated & $602(23.9)$ \\
\hline Q4 [81+] & $665(24.9)$ & Histology & \\
\hline Mean (Std) [IQR] & $74.8(8.4)[11]$ & Tube/colloid & $138(5.2)$ \\
\hline Rural Status & & Other & $74(2.8)$ \\
\hline Metro & $1584(59.2)$ & Ductal/lobular & $2463(92.1)$ \\
\hline Urban & $967(36.2)$ & Lymph Nodes & \\
\hline Rural & $124(4.6)$ & Positive & $413(15.4)$ \\
\hline Singh Index & & Negative & $2262(84.6)$ \\
\hline Q1 [Highest SES] & $687(25.7)$ & ER/PR positive & $N_{\text {miss }}=99$ \\
\hline Q2 & $674(25.2)$ & Positive & $2264(87.9)$ \\
\hline Q3 & $649(24.3)$ & Negative & $312(12.1)$ \\
\hline Q4 [Lowest SES] & $665(24.9)$ & Tumor Size & $N_{\text {miss }}=88$ \\
\hline Mean (Std) [IQR] & 86.3 (13.3) [16] & $\mathrm{Q} 1[\leq 9 \mathrm{~mm}]$ & $708(27.4)$ \\
\hline Access to Near Radiation Facilities & & Q2 [10-13] & $601(23.2)$ \\
\hline Q1 [ $\leq 2]$ & $1056(39.5)$ & Q3 [14-20] & $727(28.1)$ \\
\hline Q2 [3-4] & $497(18.6)$ & Q4 [20+] & $551(21.3)$ \\
\hline Q3 [5] & $530(19.8)$ & Mean(Std) $[\mathrm{IQR}]$ & $15.7(11.5)[11]$ \\
\hline Q4 [6+] & $592(22.1)$ & COC status & \\
\hline Mean (Std) [IQR] & $4.7(4.7)[3]$ & Yes & $1898(71.0)$ \\
\hline Region & & No & $777(29.1)$ \\
\hline Kentucky & $251(9.4)$ & SF Number of Beds & $N_{\text {miss }}=32$ \\
\hline North Carolina & $504(18.8)$ & Mean $(\mathrm{Std})[\mathrm{IQR}]$ & 339 (244) [279] \\
\hline Ohio & $525(19.6)$ & SF Radiation Services & $\mathrm{N}_{\text {miss }}=45$ \\
\hline Pennsyvlania & $1395(52.2)$ & Yes & $2031(77.2)$ \\
\hline Marital Status & $N_{\text {miss }}=72$ & No & $599(22.8)$ \\
\hline Single & $1390(53.4)$ & Optional RT & $\mathrm{N}_{\text {miss }}=176$ \\
\hline Married & $1213(46.6)$ & Yes & $1098(43.9)$ \\
\hline Charlson Comorbidity Score & & No & $1401(56.1)$ \\
\hline 0 & $1014(37.9)$ & Surg Provider Volume & $\mathrm{N}_{\text {miss }}=134$ \\
\hline 1 & $805(30.1)$ & Mean $(\mathrm{Std})[\mathrm{IQR}]$ & $16.2(20.3)[20]$ \\
\hline $2+$ & $856(32.0)$ & Adjuvant Radiation Therapy & \\
\hline Meand (Std) [IQR] & $1.34(1.62)[2]$ & Yes & $2128(79.6)$ \\
\hline Medicaid Dual & & No & $547(20.5)$ \\
\hline Yes & $387(14.5)$ & OS Survival in months ${ }^{\S \S}$ & \\
\hline No & $2288(85.5)$ & Observed Mean & $5.5(1.9)[2.0]$ \\
\hline \multicolumn{4}{|l|}{ Institutionalized } \\
\hline Yes & $163(6.1)$ & & \\
\hline No & $2512(94.9))$ & & \\
\hline
\end{tabular}


Table 1 Characteristics and Predictors of Adjuvant Radiation Therapy ( $N=2675)$ (Continued)

\begin{tabular}{ll}
\hline Overall & $N(\%)$ \\
\hline Stage & \\
Stage I & $1869(69.9)$ \\
Stage II & $697(26.1)$ \\
Stage III & $109(4.1)$ \\
First Cancer & \\
Yes & $2285(85.4)$ \\
No & $390(14.6)$ \\
\hline
\end{tabular}

cohorts. The AFT curves are presented by dashed lines and KM curves are continuous. As a diagnostic of goodness of fit, the model-based unadjusted curves closely track the KM curves. Unadjusted 5 year OS rates were $60 \%$ vs $85 \%$, compared to adjusted OS rates of $71 \%$ vs $83 \%$, for those receiving RT after BCS vs BCS alone.

\section{Discussion}

In a typical population based patterns of breast cancer care study, we quantified significant confounding of the association between use of RT after BCS and survival. Confounding variables accounted for approximately half of the survival benefit of having received RT after BCS vs not. This large magnitude of confounding warrants caution in interpretation of treatment benefits in population-based studies. While the specific set of confounders that an investigator chooses to examine will depend upon various study attributes, and could differ from ours, we nonetheless sought to test a set of plausible confounders relevant to health

\begin{tabular}{|c|c|c|c|c|c|c|}
\hline & AFT Parameter Estimates and $95 \% \mathrm{Cl}$ & Ref & $\%$ Change & LCL & UCL & fdr pval \\
\hline Adj Radiation & $\longmapsto$ & & 62.56 & 41.83 & 86.32 & * \\
\hline Age: & $\longmapsto$ & Q4 & 131.2 & 88.50 & 183.6 & * \\
\hline Age: & $\longmapsto$ & Q4 & 83.84 & 55.78 & 117.0 & * \\
\hline Age: $\quad$ Q3 & $\longmapsto$ & Q4 & 49.51 & 28.06 & 74.54 & * \\
\hline Rurality: Metro & $\longmapsto$ & Rural & 29.24 & -4.39 & 74.68 & \multirow[b]{2}{*}{ * } \\
\hline Rurality: Urban & $\longmapsto$ & Rural & 47.10 & 10.82 & 95.26 & \\
\hline Singh: Hi SES Q1 & $\longmapsto$ & Low SES Q4 & $4 \quad 14.99$ & -8.80 & 45.00 & \\
\hline Singh: & $\longmapsto$ & Low SES Q4 & $4 \quad 18.08$ & -4.27 & 45.64 & \\
\hline Singh: $\quad \mathrm{Q}_{3}$ & $\longmapsto$ & LOW SES Q4 & $4 \quad 17.70$ & -1.50 & 40.65 & \\
\hline Access: Low Q1 & $\longmapsto$ & Hi Q4 & -11.4 & -28.8 & 10.10 & \\
\hline Access: $\quad$ Q2 & $\longmapsto$ & Hi Q4 & -1.40 & -20.6 & 22.44 & \\
\hline Access: $\quad$ Q3 & $\longmapsto$ & $\mathrm{HiQ4}$ & -16.4 & -30.8 & 1.04 & \\
\hline Dx State: $\quad \mathrm{KY}$ & $\longmapsto$ & PA & 19.56 & -6.46 & 52.81 & \\
\hline Dx State: NC & $\longmapsto$ & PA & -2.07 & -17.7 & 16.56 & \\
\hline Dx State: $\quad \mathrm{OH}$ & $\longmapsto$ & PA & -17.5 & -29.5 & -3.50 & * \\
\hline Single: $Y$ & $\longmapsto$ & Married & -9.17 & -20.1 & 3.21 & \\
\hline Charlson: & $\longmapsto$ & $2+$ & 82.39 & 57.98 & 110.6 & * \\
\hline Charlson: 1 & $\longmapsto$ & $2+$ & 38.81 & 21.08 & 59.14 & * \\
\hline \multirow{2}{*}{$\begin{array}{l}\text { Medicaid Dual: } Y \\
\text { In Institution: } Y\end{array}$} & $\longmapsto$ & $\mathrm{N}$ & -19.7 & -32.0 & -5.22 & * \\
\hline & \multirow[b]{2}{*}{$\longmapsto$} & $\mathrm{N}$ & -51.1 & -60.3 & -39.8 & * \\
\hline Stage: $\quad 1$ & & IIII & 27.23 & -18.3 & 98.17 & \\
\hline Stage: II & $\longmapsto$ & III & 38.89 & 5.42 & 82.99 & * \\
\hline First Cancer: $Y$ & $\longmapsto$ & $\mathrm{N}$ & 23.47 & 6.25 & 43.48 & * \\
\hline Grade: Well diff & $\longmapsto$ & Poor/Undiff & 8.34 & -10.2 & 30.78 & \\
\hline Grade: Mod. diff & $\longmapsto$ & Poor/Undiff & 8.36 & -7.47 & 26.89 & \\
\hline Hist: Ductal/Lob & $\longmapsto$ & Tube/colloid & 21.44 & -4.94 & 55.15 & \\
\hline Hist: Other & $\longmapsto$ & Tube/colloid & 28.57 & -13.4 & 90.89 & \\
\hline Lymph Nodes: Positive & $\longmapsto$ & Negative & -21.9 & -38.7 & -0.37 & \\
\hline ER/PR : Positive & $\longmapsto$ & Negative & 30.59 & 5.75 & 61.26 & * \\
\hline Tumor Size: Q1 & $\longmapsto$ & Q4 & 83.49 & 37.02 & 145.7 & * \\
\hline Tumor Size: Q2 & $\longrightarrow$ & Q4 & 60.30 & 20.52 & 113.2 & * \\
\hline Tumor Size: Q3 & $\longmapsto$ & Q4 & 49.20 & 13.90 & 95.45 & * \\
\hline No COC Status: $Y$ & $\longmapsto$ & $N$ & -5.71 & -17.9 & 8.31 & \\
\hline$\#$ of Beds Trend & $\longmapsto$ & & 9.48 & 1.00 & 18.66 & \\
\hline Provider Volume Trend & $\mapsto-1$ & & -0.06 & -5.83 & 6.05 & \\
\hline RT Facility Services & $\longmapsto$ & $\mathrm{N}$ & -7.76 & -20.6 & 7.16 & \\
\hline RT optional : Y & $\longmapsto$ & $\mathrm{N}$ & -7.03 & -24.1 & 13.91 & \\
\hline & OS Survival Less Likely & & & & & \\
\hline-100 & 100 & & & & & \\
\hline $\begin{array}{l}\text { Note: } \text { Ref = Reference, \% Chang } \\
\text { Estimates are based on main ef }\end{array}$ & $\begin{array}{l}\text { ge }=\% \text { Change in expected survival, } L C L, U C L=95 \% \text { Confid } \\
\text { ffects multivariate model. }\end{array}$ & e Intervals & ls, fdr $p$ & $=F$ & djuste & p-value; \\
\hline $\begin{array}{l}\text { Fig. } 2 \text { Predictors of OS survival. } \\
\text { overall survival }\end{array}$ & Shows parameter estimates for AFT multivariate model whe & adjuvant ra & radiation ar & id othe & covaria & es predict \\
\hline
\end{tabular}



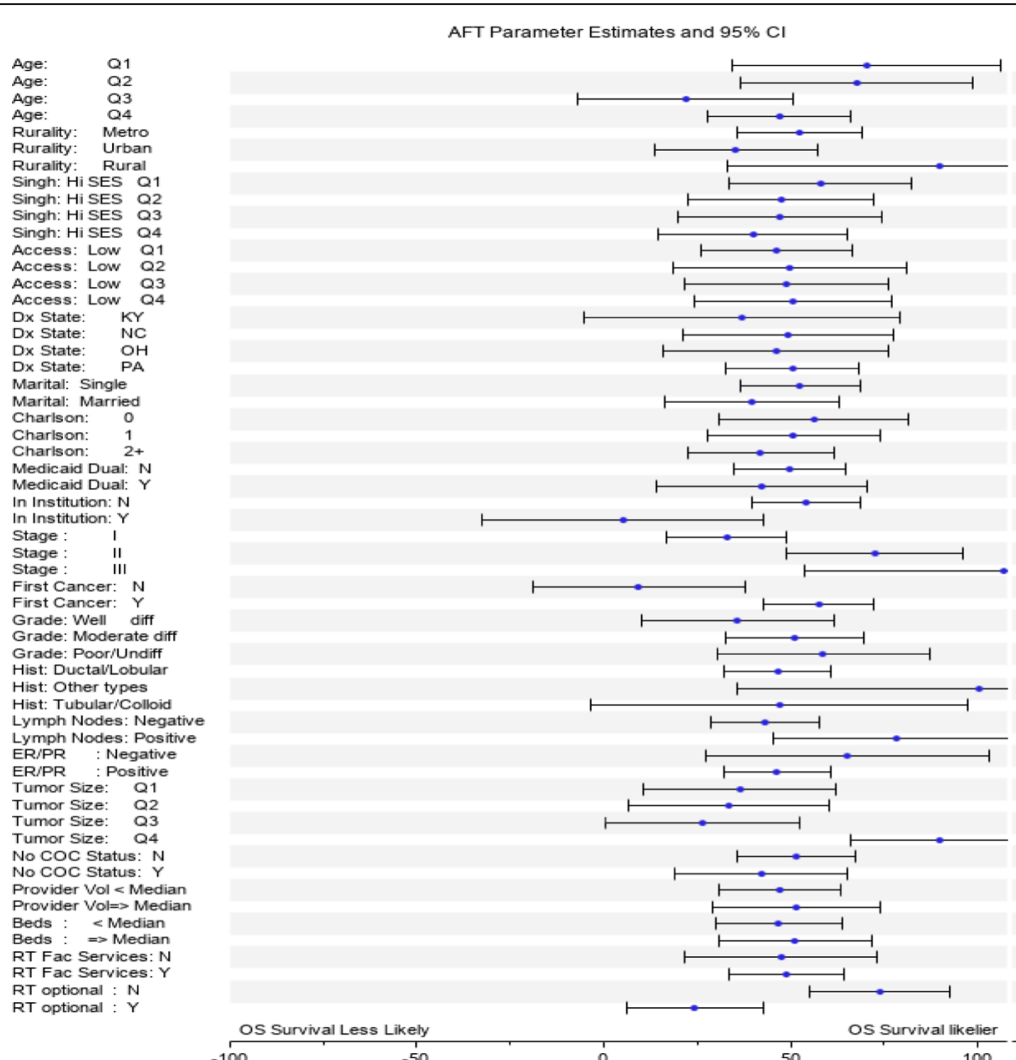

\begin{tabular}{|c|c|c|c|}
\hline Change & LCL & UCL & Int $p$ \\
\hline $\begin{array}{l}70.4 \\
67.8\end{array}$ & $\begin{array}{l}34.6 \\
36.7\end{array}$ & $\begin{array}{r}106.2 \\
98.8\end{array}$ & 0.0947 \\
\hline 21.8 & $\begin{array}{l}30.7 \\
-6.9\end{array}$ & 50.5 & \\
\hline 47.0 & 27.8 & 66.2 & \\
\hline $\begin{array}{l}52.3 \\
35.4\end{array}$ & $\begin{array}{l}35.5 \\
13.7\end{array}$ & $\begin{array}{l}69.2 \\
57.2\end{array}$ & 0.1536 \\
\hline 90.1 & 32.8 & 147.3 & \\
\hline $\begin{array}{l}58.1 \\
47.5\end{array}$ & $\begin{array}{r}33.7 \\
22.5\end{array}$ & $\begin{array}{l}82.5 \\
72.4\end{array}$ & 0.7732 \\
\hline 47.0 & 19.7 & 74.3 & \\
\hline 39.9 & 14.7 & 65.1 & \\
\hline 46.2 & 26.0 & 66.3 & 0.9932 \\
\hline 49.8 & 18.4 & 81.2 & \\
\hline 48.9 & 21.6 & 76.3 & \\
\hline 50.6 & 24.2 & 76.9 & \\
\hline 37.1 & -5.2 & 79.4 & 0.9473 \\
\hline $\begin{array}{l}49.4 \\
46.1\end{array}$ & $\begin{array}{l}21.2 \\
16.0\end{array}$ & $\begin{array}{l}77.5 \\
76.2\end{array}$ & \\
\hline 50.5 & 32.5 & 68.5 & \\
\hline 52.6 & 36.5 & 68.7 & 0.3784 \\
\hline $\begin{array}{l}39.7 \\
562 .\end{array}$ & $\begin{array}{l}16.4 \\
31.0\end{array}$ & $\begin{array}{l}63.1 \\
81.4\end{array}$ & 0.6404 \\
\hline 50.7 & 27.7 & 73.8 & \\
\hline 41.9 & 22.4 & 61.5 & 06290 \\
\hline $\begin{array}{l}49.9 \\
42.3\end{array}$ & 34.8 & 64.9 & 0.6290 \\
\hline $\begin{array}{l}42.3 \\
54.1\end{array}$ & $\begin{array}{l}14.2 \\
39.6\end{array}$ & $\begin{array}{l}70.3 \\
68.5\end{array}$ & 0.0160 \\
\hline 5.2 & $\begin{array}{r}39.0 \\
-32.4\end{array}$ & 42.7 & 0.0100 \\
\hline 32.8 & 16.6 & 49.0 & 0.0016 \\
\hline 72.6 & 49.0 & 96.1 & 0.0010 \\
\hline 107.2 & 53.7 & 160.6 & \\
\hline 9.4 & -18.9 & 37.8 & 0.0022 \\
\hline 57.6 & 42.9 & 72.3 & \\
\hline 35.8 & 10.2 & 61.5 & 0.4101 \\
\hline $\begin{array}{l}51.1 \\
58.7\end{array}$ & $\begin{array}{l}32.5 \\
30.3\end{array}$ & 69.8 & \\
\hline $\begin{array}{l}58.7 \\
46.5\end{array}$ & $\begin{array}{l}30.3 \\
32.3\end{array}$ & $\begin{array}{l}87.0 \\
607\end{array}$ & 0.2729 \\
\hline 100.6 & 35.8 & 165.5 & \\
\hline 47.0 & -3.5 & 97.5 & \\
\hline 43.2 & 28.5 & 57.8 & 0.0506 \\
\hline 78.3 & 45.4 & 111.2 & \\
\hline 65.1 & 27.1 & 103.1 & 0.3715 \\
\hline $\begin{array}{l}46.4 \\
36.4\end{array}$ & $\begin{array}{l}32.0 \\
10.5\end{array}$ & $\begin{array}{l}60.8 \\
62.2\end{array}$ & 0.0005 \\
\hline 33.5 & 6.7 & 60.2 & \\
\hline 26.5 & 0.6 & 52.5 & \\
\hline 90.0 & 66.1 & 113.9 & \\
\hline 51.4 & 35.5 & 67.4 & 0.4959 \\
\hline 42.2 & 19.0 & 65.3 & \\
\hline 47.2 & 30.7 & 63.6 & 0.8063 \\
\hline $\begin{array}{l}51.4 \\
46.9\end{array}$ & $\begin{array}{l}28.9 \\
300\end{array}$ & $\begin{array}{l}74.0 \\
63.8\end{array}$ & 0.7477 \\
\hline $\begin{array}{l}46.9 \\
51.3\end{array}$ & $\begin{array}{l}30.0 \\
30.8\end{array}$ & $\begin{array}{l}63.8 \\
71.8\end{array}$ & \\
\hline 47.5 & 21.8 & 73.3 & 0.9119 \\
\hline 48.9 & 33.6 & 64.3 & \\
\hline 73.8 & 55.0 & 92.6 & $<.0001$ \\
\hline 24.4 & 6.0 & 42.7 & \\
\hline
\end{tabular}

Note: $\%$ Change $=\%$ Change in expected survival between RT vs no RT, LCL, UCL $=95 \%$ Confidence Intervals; Int $p=$ Wald Test of interaction, or, differences in effects within group.

Fig. 3 Adjuvant Radiation Estimates by subgroup analysis. Shows parameter estimates for subgroup analysis

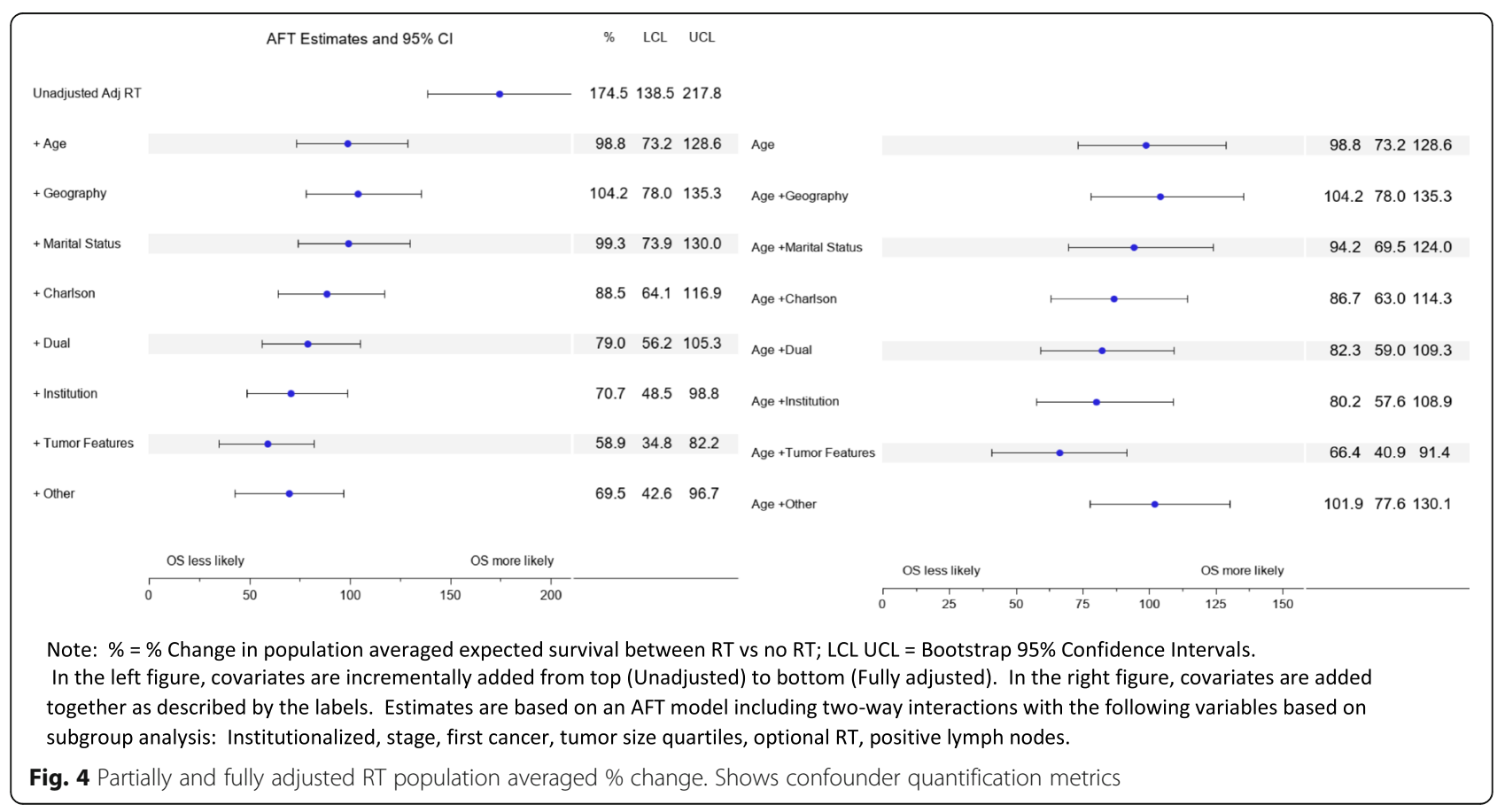


Probability of Survival

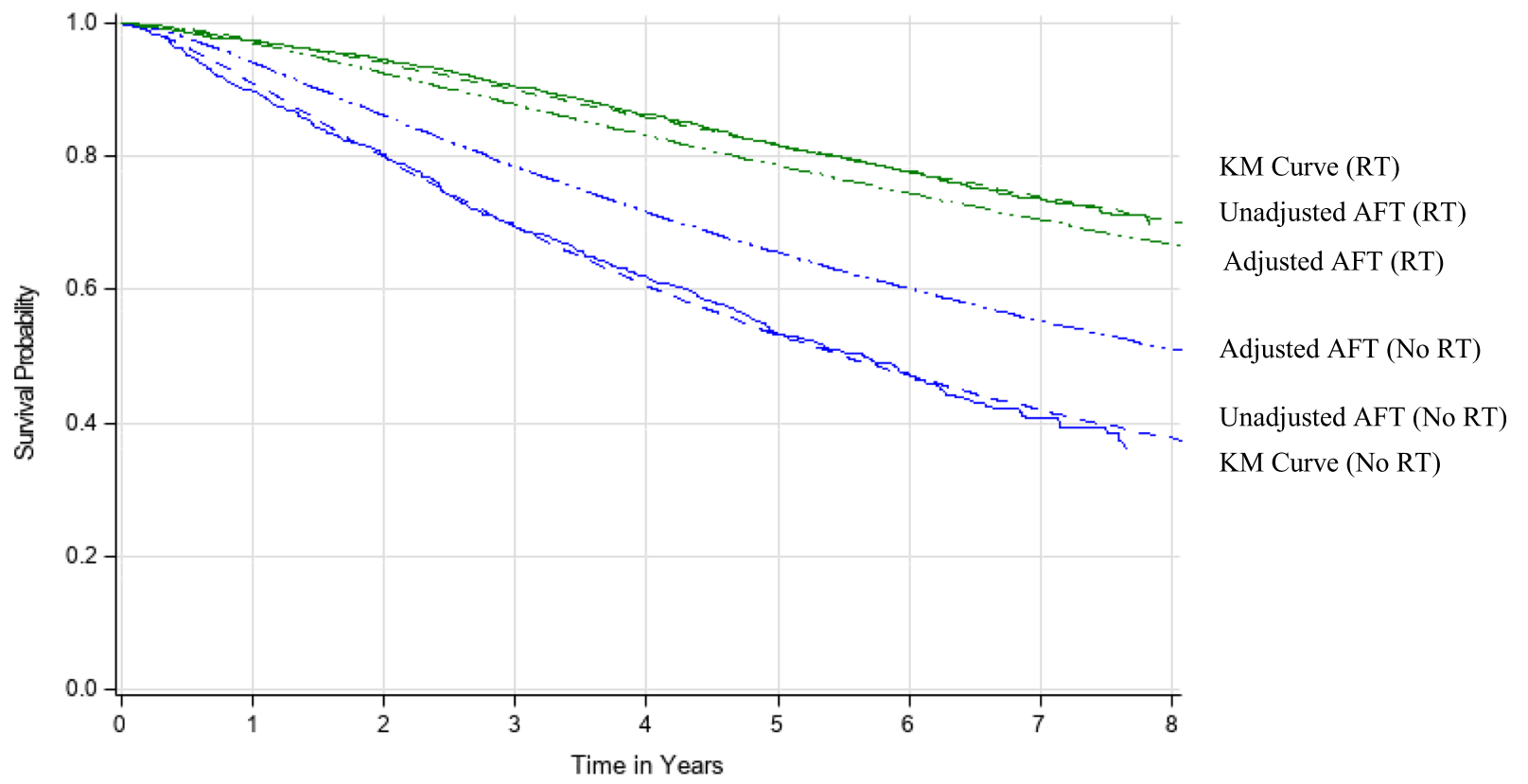

No Adjuvant RT

Adjuvant RT

Note: Sample is restricted to beneficiaries who survived 1 year after diagnosis and survival time starts 1 year after diagnosis.

Fig. 5 Overall Survival Curves. Shows adjusted and unadjusted survival curves

services research and health disparities in breast cancer treatment in an elderly sample.

While adjusting for confounding factors in analyzing the effect of BCS RT on survival is standard practice, there are relatively few modern papers in oncology and health disparities that examine and demonstrate the magnitude of this source of bias. Prior studies have hypothesized on possible mechanisms which may account for unanticipated higher mortality rates in women who forego RT after BCS, ranging from comorbidity, poverty, and lack of access [11], with particular suspicion centering on comorbidity and disability [23]. Our analysis suggests that, while comorbidity and associated measures may contribute towards reducing confounding bias incrementally and individually, adjusting for age, and tumor characteristics only as performed in most epidemiologic and randomized studies [46-48] may account for most of the bias (66\% change vs $70 \%$ change, $4 \%$ bias, Fig. 4 ).

Age significantly had the most contribution to confounding (71\%). Although this finding may not be noteworthy given that age is a standard covariate in survival analysis, the unexpected magnitude of contribution suggests unadjusted RT survival curves may be subject to substantial bias if not controlled for age.

Appalachian regions varied considerably by sociodemographic characteristics. Most notably, patients in
KY lived in significantly more rural areas than PA (20\% vs $1 \%, p<.0001$, using county-level USDA Beale codes) with the average number of nearby radiation facilities within a 50-mile straight-line radius of patient residence in $\mathrm{KY}$ being significantly lower than PA (1.31 vs 6.2, $p<.0001)$, and with significantly lower regional SES $(p<.0001)$ as well. Patients living in KY and NC were significantly less likely than PA to receive RT by approximately 6 percentage points. However, the addition of geographic variables to the age adjusted model resulted in an increase in the association of RT on OS. This result is explained by a pattern of better survival in KY compared to other states. For example, a percent change in survival of $20.6 \%$ vs $\mathrm{PA}, 46.0 \%$ vs $\mathrm{OH}$, and $22.7 \%$ vs NC was observed under the main effects model. This was unexpected given KY's rurality. After adjusting for state of residence, the other geographical measures, including health care access, SES (Socioeconomic Status), and rurality, are not related to receipt of RT and thus do not contribute to incremental confounding.

Consistent with comorbidity being hypothesized as an important confounder [10], its contribution to incremental confounding ranks was positive (11\%) although not as strong as we expected. Confounding may occur because higher comorbidity coincides with increased risk of death and at the same time women who have more 
comorbidity are less likely to receive RT. Furthermore, this contribution may be stronger than in other samples given that the generally higher rates of comorbidity in rural Appalachia than more affluent areas of the United States may increase the importance of this source of confounding [49, 50]. Alternatively, it is possible the Charlson-comorbidity score does not fully capture a patient's level of illness unrelated to cancer as similar samples have reported lower rates of no-comorbidity [51].

A related health status confounder was institutionalization assessed as ongoing care in a skilled nursing facility or receipt of domiciliary care. While few population-based patterns of care studies include measures of this status in their models, our findings suggest that it is a potential source of confounding with regard to access to cancer treatment and survival (8\% in incremental confounding using default order, $18 \%$ when age is included only, and $90 \%$ jointly with age). Institutionalized breast cancer patients may suffer from increased barriers to treatment and should be considered in multivariate models comparing treatments or populations.

Medicaid dual status, as an indicator of means-tested poverty, also contributed to reduction of confounding bias. The study region includes extremes of highly concentrated poverty in isolated rural areas of Appalachia to more affluent regions in PA and urbanized areas. The effect of poverty on access to breast cancer care and on outcomes is well documented in the literature [52-54] and is commonly included, along with age, in analytical models. The fully adjusted model included both area poverty (Singh index) and individual-level poverty assessed by dual status; both predicted access to RT (not shown) but only the latter was a significant source of confounding in the relationship of RT to OS.

We considered including concomitant treatments, such as use of adjuvant endocrine therapy (AET) in the survival model, but made the decision not to for three main reasons. First, the analysis would have been limited by the availability of pharmacy information for only two thirds of the sample. Secondly, it was unclear in our estimation whether such treatments are confounders, along confounding pathways, or mediators of the association between RT and survival, in which case the fully adjusted effect would be an over-controlled effect. Lastly, in the case of AET, such therapy is restricted primarily to ER/PR positive tumors and was not found to be predictive of survival in this stratum after fully adjusting for the other variables ( $\%$ change $=13$, Not Significant), possibly due to small sample size, low-risk observational populations, and too short follow-up to observe survival benefit.

The quantification approach assumes the data generating mechanism is a linear main effect AFT model with potential two-way interactions between exposure (RT) and covariates, which is a common assumption made when modelling using regression analysis. In addition failure to satisfy the backdoor criterion when conditioning on confounders may bias the estimates and may occur as a result of measurement error in the exposure/covariates or presence of hidden latent confounders. Furthermore, incremental confounding contributions may change depending on the order of decomposition, though we think our order is desirable as it follows antecedent causality (i.e. age affects comorbidity and not vice-versa).

Informally, we view the strength of confounding as a product of the association between the confounder with receipt of RT and its association with survival. Although the generalizability of the decomposition in other samples may be affected due to variations in these associations, we hypothesize the relative ordering and magnitudes will not be substantial in population based samples, such as this one involving elderly beneficiaries in the United States. In such is the case, findings and implications will then be applicable to similar settings.

\section{Conclusions}

We explored the contribution of confounding variables to the unexpected, short-term OS benefit of the addition of $\mathrm{RT}$ to BCS versus BCS alone seen in epidemiologic studies. Quantification of confounding aids in determining covariates to include in a multivariate model and in interpreting raw associations. Substantial confounding was present (60\% of total association), with age accounting for the largest share (71\%); adjusting for age plus tumor features corrected for most of the confounding, resulting in only a $4 \%$ bias. The direct effect of having received RT after BCS on OS, however, seemed to account for $40 \%$ of the benefit.

\section{Supplementary information}

Supplementary information accompanies this paper at https://doi.org/10. 1186/s12885-019-6263-3.

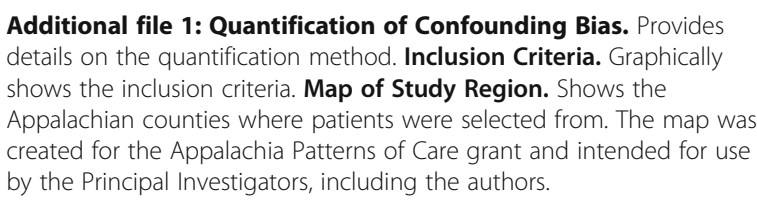

Additional file 1: Quantification of Confounding Bias. Provides details on the quantification method. Inclusion Criteria. Graphically shows the inclusion criteria. Map of Study Region. Shows the Appalachian counties where patients were selected from. The map was created for the Appalachia Patterns of Care grant and intended for use by the Principal Investigators, including the authors.

\footnotetext{
Abbreviations

$\%$ CH: Percent change; AET: Adjuvant Endocrine Therapy; AFT: Accelerated Failure Time; AHA: American Hospital Association; AIC: Akaike Information Criterion; AJCC: American Joint Committee on Cancer; BCS: Breast conserving surgery; COC: Commission of Cancer; CPT: Current Procedural Terminology; DAGS: Directed Acyclic Graphs; E[T]: Expected survival time; ER/PR: Estrogen/ Progesterone Receptor; FCS: Fully Conditional Specification; FFS: Fee for Service; HCPCS: Healthcare Common Procedure Coding System; IC: Incremental Confounding; ICD-9: International Classification of Diseases, Ninth Revision, Clinical Modification; IPTW: Inverse Probability of Treatment Weights; KM: Kaplan Meier; KY: Kentucky; MI: Multiple Imputation; NC: North Carolina; NCl: National Cancer Institute; NDI: National Death Index; NS: Not Significant; OH: Ohio; OS: Overall Survival; PA: Pennsylvania; RT: Radiation therapy; SAS: Statistical Analysis System; SEER: Surveillance, Epidemiology,
} 
and End Results Program; SES: Socioeconomic Status; USDA: US Department of Agriculture

\section{Acknowledgements \\ Not applicable.}

\section{Authors' contributions}

TFC, RA and GK have made substantial contributions to design/analysis/ interpretation/drafting of this manuscript. Mr. TFC is responsible for the statistical methods used in this study. All authors have read and approved the final manuscript.

\section{Funding}

This study was funded by NCl Patterns of Care in Appalachia, Grant \#1R01CA140335-01A1. This study was supported entirely with funds provided by the UVA Cancer Center (UVACC) to Dr. Anderson for research program development. Dr. Anderson serves as Associate Director of Population Sciences and is a co-author of this paper as a scientific investigator. The UVACC did not otherwise influence the design or conduct of this study.

\section{Availability of data and materials}

The datasets generated and analyzed during the current study are not publicly available due privacy restrictions placed by the data supplier (CMS, Centers for Medicare and Medicaid Services) and agreements with local registries. Please contact the first author F. Camacho for further details and access to the data.

\section{Ethics approval and consent to participate}

This study satisfied conditions for institutional IRB waiver of informed consent through exemption (University of Virginia Institutional Review Board for Health Sciences Research, tracking ID\# 19628). Administrative approval to access the study data by the study team was granted by CMS under a Data Use Agreement with the University of Virginia.

\section{Consent for publication}

Not applicable.

\section{Competing interests}

The authors declare that they have no competing interests.

\section{Author details}

${ }^{1}$ Department of Public Health Sciences, University of Virginia, Charlottesville, VA 22903, USA. ²Duke Cancer Institute, Duke University, Durham, USA.

Received: 19 March 2019 Accepted: 15 October 2019 Published online: 17 December 2019

\section{References}

1. Darby S, McGale P, Correa C, Taylor C, Arriagada R, Clarke M, Cutter D, Davies C, Ewertz M, Godwin J, Gray R, Pierce L, Whelan T, Wang Y, Peto R. Effect of radiotherapy after breast-conserving surgery on 10-year recurrence and 15-year breast cancer death: meta-analyis of individual patient data for 10,801 women in 17 randomized trials. Lancet. 2011;378(9804):1707-16.

2. Clarke M, Collins R, Darby S, Davies C, Ephinstone P, Evans E, Godwin J, Gray R, Hicks C, James S. Effects of Radiotherapy and Difference in the Extent of Surgery for Early Breast Cancer on Local Recurrence and 15-Year Survival: An Overview of the Randomised Trials. Lancet. 2005;366(9503):2087.

3. Vinh-Hung $V$, Verschraegen $C$. Breast-conserving surgery with or without radiotherapy: pooled-analysis for risks of ipsilateral breast tumor recurrence and mortality. J Natl Cancer Inst. 2004;96:115-21.

4. Vallis K, Tannock I. Postoperative radiotherapy for breast Cancer: growing evidence for an impact on survival. J Natl Cancer Inst. 2004;96(2):88-9.

5. Eifel P, Axelson J, Costa J, Crowley J, Curran W, Deshler A, Fulton S, Hendricks C, M K, Kornblith A, Louis T, Markman M, Mayer R, Roter D. National Institutes of Health consensus development conference statement: adjuvant therapy for breast cancer. J Natl Cancer Inst. 2001;93(13):979-89.

6. Guy G, Lipscomb J, Gillespie T, Goodman M, Richardson L, Ward K. Variations in Guideline-Concordant Breast Cancer Adjuvant Therapy in Rural Georgia. Health Serv Res. 2014;50(4):1088.
7. Anderson R, Morris C, Kimmick G, Trentham-Dietz A, Camacho F, Wu X. Patterns of locoregional treatment for nonmetastatic breast cancer by patient and health system factors. Cancer. 2015;121(5):790-9.

8. Vinh-Hung V, Burzykowski T, Van de Steen J, Storme G, Soete G. Postsurgery radiation in early breast cancer: survival analyis of registry data. Radiotherapy Ongolocy. 2002;64:281-90.

9. Vinh-Hung V, Voordeckers M, Van de Steen J, Soete G, Lamot J, Storme G. Omission of radiotherapy after breast-conserving surgery: survival impact and time trends. Radiother Oncol. 2003;67:147-58.

10. Kimmick G, Camacho F, Hwang W, Mackley H, Stewart J, Anderson R. Adjuvant radiation and outcomes after breast conserving surgery in publicly insured patients. J Geriatr oncol. 2012;3(2):138-46.

11. Foley K, Kimmick G, Camacho F, Levine E, Balkrishnan R, Anderson R. Survival disadvantage among Medicaid-insured breast cancer patients treated with breast conserving surgery without radiation therapy. Breast Cancer Res Treat. 2007;101(2):207-14.

12. Dragun A, Huang B, Tucker T, Spanos W. Disparities in the application of adjuvant radiotherapy after breast-conserving surgery for early stage breast cancer. Cancer. 2010;117(12):2590-8.

13. Janes $H$, Dominici $F$, Zeger S. On quantifying the magnitude of confounding. Biostatistics. 2010;11(3):572-82.

14. Halverson J, Bichak G. Underlying socioeconomic factors influencing health disparities in the Appalachia region: final report. Washington, DC: Appalachian Regional Commission; 2008.

15. Wingo $P$, Tucker $T$, Jamison $P$, Martin H, McLaughlin C, Bayakly R, BolickAldrich S, Colsher P, Indian R, Knight K, Neloms S, Wilson R, Richards T. Cancer in Appalachia 2001-2003. Cancer. 2008;112:181-92.

16. Lengerich E, Tucker T, Powell R, Colsher P, Lehman E, Ward A, Siedlecki J, Wyatt S. Cancer incidence in Kentucky, Pennsylvania, and West Virginia: disparities in Appalachia. J Rural Health. 2005;21:39-47.

17. Yao N, Lengerich $E$, Hillemeier M. Breast cancer mortality in Appalachia: reversing patterns of disparity over time. J Health Care Poor Underserved. 2012;23(2):715-25.

18. Fleming S, Mackley H, Camacho F, Seiber E, Gusani J, Matthews S, Liao J, Yang T, Hwang W, Yao N. Clinical, socio-demographic, and service provider determinants of guideline concordant colorectal cancer care for Appalachian residents. J Rural Health. 2014;30(1):29-37.

19. Lane N, Lutz A, Baker K, Konrad T, Ricketts T, Randolph R, Tran C, Beadles C. "Health care costs and access disparities in Appalachia," PDA Inc, and Cecil B Sheps Center for Health Services Research. North Carolina: Chapel Hill; 2012

20. Du X, Freeman J, Goodwin J. Information on radiation treatment in patients with breast cancer: the advantages of the linked Medicare and SEER data. J Clin Epidemiol. 1999;52:463-70.

21. Du X, Freeam J, Nattinger A, Goodwin J. Survival of women after breast conserving surgery for early stage breast cancer. Breast Cancer Res Treat. 2002;72:23-31.

22. Brooks J, Chrischilles E, Scott S, Ritho J, Chen-Hardee S. Information gained from linking SEER cancer registry data to state-level hospital discharge abstracts. Med Care. 2000;38:1131-40.

23. Kimmick G, Camacho F, Mackley H, Kern T, Yao N, Matthews S, Fleming S, Lipscomb J, Liao J, Hwang W, Anderson R. Individual, area, and provider characteristics associated with care received for stages I-III breast cancer in a multi-state region of Appalachia. J Oncol Pract. 2015;11(1):e9-e18. https:// www.ncbi.nlm.nih.gov/pmc/articles/PMC4295425/.

24. Cancer Research Network, "Cancer Look-up Tables," Cancer Research Network, 2018. [Online]. Available: https://crn.cancer.gov/resources/codes. html. Accessed Nov 2018.

25. Economic Research Service, "Rural-Urban Continuum Codes," US Department of Agriculture, 2018. [Online]. Available: https://www.ers. usda.gov/data-products/rural-urban-continuum-codes/. Accessed Nov 2018.

26. Singh G. Deprivation and widening inequalities in US mortality, 1969-1998 Am J Public Health. 2003;93(7):1137-43.

27. American Hospital Assoication. AHA annual survey database. Ann Arbor: American Hospital Association; 2010.

28. Deyo R, Cherkin D, Ciol M. Adapting a clinical comorbidity index for use with ICD-9-CM administrative databases. J Clin Epidemiol. 1992;45:613-9.

29. Romano $P$, Roos $L$, Jollis J. Adapting a clinical comorbidity index for use with ICD-9-CM administrative data: differing perspectives. J Clin Epidemiol. 1993;46:1075-9. 
30. American College of Surgeons, "Cancer programs," 2018. [Online]. Available: http://www.facs.org/cancerprogram/index.html. Accessed 2011.

31. Tinterri C, Gatzemeier W, Zanini V, Regolo L, Pedrazzoli C, Rondini E, Amanti C, Gentile G, Taffurelli M, Fenaroli P, Tondini C, Sacchetto G, Sismondi P, Murgo R, Orlandi M, Cianchetti E, Andreoli C. Conservative surgery with and without radiotherapy in elderly patients with early-stage breast cancer: a prospective randomized multicentre trial. Breast. 2009;18(6):373-97.

32. Ford H, Coombes R, Gazet J, Gray R, McConkey C, Sutcliffe R, Quilliam J, Lowndes S. Long-term follow-up of a randomized trial designed to determine the need of irradiation following conservative surgery the treatment of invasive breast cancer. Ann Oncol. 2006;17(3):401-8.

33. Funk M, Westreich D, Wiesen C, Sturmer T, Brookhart M, Davidian M. Doubly robust estimation of causal effects. Am J Epidemiol. 2011;173(7):761-7.

34. Elwert F. "graphical causal models," in Handbook of Causal Analysis for Social. New York: Sage Publications; 2013. p. 245-73.

35. Swindell W. Accelerated failure time models provide a useful statistical framework for aging research. Exp Gerontol. 2009:44(3):190-200.

36. Zare A, Hosseini M, Mahmoodi M, Mohammad K, Zeraati H, Holakouie N. A comparison between accelerated failure-time and cox proportional hazards modles in analyzing the survival of gastric cancer patients. Iran J Public Health. 2015;44(8):1095-102.

37. Zhang D, Tsiatis A. Analysis of Survival Data ST745 Chapter 5. 2005. Available: https://www4.stat.ncsu.edu/ dzhang2/st745/index.html. Accessed Nov 2018.

38. Collett D. Modeling survival data in Medicare research (2nd ed). London: Chapman \& Hall; 2003

39. Benjamini Y, Hochberg Y. Controlling the false discovery rate: a practical and powerful approach to multiple testing. J R Stat Soc Ser B. 1995;57(1): 289-300.

40. Klein J, Moeschberger M. "chapter 12". In: Survival analysis: techniques for censored and truncated data. New York: Springer; 2003.

41. Chang I, Gelman R, Pagano M. Corrected group prognostic curves and summary statistics. J Chronic Dis. 1982;35:669-74.

42. Makuch R. Adjusted survival curve estimation using covariates. J Chronic Dis. 1982;35:437-43.

43. Gail M, Byar D. Variance calculations for direct adjusted survival curves, with applications to testing for no treatment effect. Biom J. 1986;28:587-99.

44. White I, Royston P. Imputing missing covariate values for the cox model. Stat Med. 2009;28(15):1982-98.

45. Schomaker M, Heumann C. Bootstrap inference when using multiple imputation. Stat Med. 2018;37(14):2252-66.

46. Smith B, Gross C, Smith G, Galusha D, Bekelman J, Haffty B. Effectiveness of radiation therapy for older women with early breast cancer. J Natl Cancer Inst. 2006:98(10):681-90.

47. Truong P, Bernstein V, Lesperance M, Speers C, Olivott I. Radiotherapy omission after breast-conserving surgeryis associated with reduced breast cancer-specific survival in elderly women with breast cancer. Am J Surg. 2006;191(6):749-55.

48. Matuschek C, Bolke E, Haussman J. The benefit of adjuvant radiotherapy after breast conserving surgery in older patients with low risk breast cancera meta-analysis of randomized trials. Radiat Oncol. 2017;12. https:/www. ncbi.nlm.nih.gov/pubmed/28335784.

49. Halverson J, Bichak G. Underlying socioeconomic factors influencing health disparities in the Appalachian region. Washington DC: Appalachian Regional Comission; 2008

50. Barker L, Crespo R, Gerzoff R, Denham S, Shrewsberry M, A.D C. Residence in a distressed county in Appalachia as a risk factor for diabetes. Behavioral Risk Factor Surveillance System, 2006-227. Prev Chronic Dis. 2010;7(5):A104.

51. Yancik R, Wesley M, Ries L, Havlik R, Edwards B, Yates J. Effect of age and comorbidity in postmenopausal breast cancer patients aged 55 years and older. JAMA. 2001:285(7):885-92.

52. Bradley C, Gardiner J, Given C, Roberts C. Cancer, Medicaid enrollment, and survival disparities. Cancer. 2005;103(8):1712-8.

53. Schrijvers C, Mackenbach J, Lutz J, Quinn M, Coleman M. Deprivation and survival from breast cancer. Br J Cancer. 1995;72(3):738-43.

54. Gentil-Brevet J, Colonna M, Danzon A, Grosclaude P, Chaplain G, Velten M, Bonnetain F, Arveux P. The influence of socio-economic and surveillance characteristics on breast cancer survival: a French population-based study. Br J Cancer. 2008;98(1):217-24.

\section{Publisher's Note}

Springer Nature remains neutral with regard to jurisdictional claims in published maps and institutional affiliations.
Ready to submit your research? Choose BMC and benefit from:

- fast, convenient online submission

- thorough peer review by experienced researchers in your field

- rapid publication on acceptance

- support for research data, including large and complex data types

- gold Open Access which fosters wider collaboration and increased citations

- maximum visibility for your research: over $100 \mathrm{M}$ website views per year

At BMC, research is always in progress.

Learn more biomedcentral.com/submissions 